\title{
Accuracy enhancing methods for the frequency-weighted balancing related model reduction
}

\author{
Andras Varga \\ German Aerospace Center \\ DLR - Oberpfaffenhofen \\ Institute of Robotics and System Dynamics \\ D-82234 Wessling, Germany \\ Andras.Varga@dlr.de
}

\author{
Brian D.O. Anderson \\ Research School of \\ Information Sciences and Engineering \\ Australian National University \\ Canberra ACT 0200, Australia \\ Brian.Anderson@anu.edu.au
}

\begin{abstract}
In this paper we develop computational techniques with enhanced accuracy for two frequency-weighted model reduction approaches, namely, balanced truncation, and singular perturbation approximation. New stability-enforcing choices of the frequency-weighted grammians can guarantee the stability of reduced models for two-sided frequency weights. Several numerical examples show the effectiveness of the new approximation techniques.
\end{abstract}

\section{Introduction}

Consider the $n$-th order stable original state-space model $\mathbf{G}:=(A, B, C, D)$ with the transfer-function matrix (TFM) $G(\lambda)=C(\lambda I-A)^{-1} B+D$, where $\lambda=s$ is the complex Laplace-transform variable in the case of a continuous-time system or $\lambda=z$ is the complex $Z$-transform variable in the case of a discrete-time system. Let $\mathbf{G}_{\mathbf{r}}:=\left(A_{r}, B_{r}, C_{r}, D_{r}\right)$ be an $r$-th order approximation of the original model $(r<n)$, with the TFM $G_{r}(\lambda)=C_{r}\left(\lambda I-A_{r}\right)^{-1} B_{r}+D_{r}$. The methods for frequency-weighted model reduction (FWMR) try to make as small as possible a weighted error of the form

$$
\left\|W_{o}\left(G-G_{r}\right) W_{i}\right\|_{\infty}
$$

where $W_{o}$ and $W_{i}$ are suitably chosen output and input weighting TFMs, respectively. The presence of weights reflects the desire that the approximation be more accurate at certain frequencies where $W_{o}$ and/or $W_{i}$ have larger singular values. Several controller reduction problems addressing closed-loop stability and performance preserving can be formulated as special one-sided or two-sided FWMR problems [1].

Reliable numerical methods for unweighted approximations are well understood and accompanying robust numerical software based on square-root and balancingfree accuracy enhancing techniques is freely available
[14]. Using these codes, dense problems up to order 1000 have been solved successfully on desktop personal computers. In this paper we extend these accuracy enhancing techniques to the frequency-weighted approximation methods related to the balancing method introduced by Enns [3].

One difficulty with the Enns' method is the lack of any guarantee of stability of the reduced model in the case of two-sided weighting. We propose here a new parametrized selection scheme of the grammians which combines the advantages of Enns' method with those of the stability guaranteeing technique proposed in [6]. Alternative stability-enforcing schemes are also proposed which offer additional flexibility in using frequency-weighting techniques with easily computable error bounds. The new schemes provide a wide range of possibilities for choosing grammians to be used for the frequency-weighted model reduction. For all choices of grammians, square-root formulas are developed. These formulas allow the usage of balancing-free square-root methods for the frequency-weighted balancing related model reduction methods.

Several numerical examples illustrate the additional gain in flexibility provided by the new selection schemes. An interesting aspect revealed in these examples is the apparently better robustness of the singular perturbation approximation method, which leads not only to lower approximation errors but also stable ones, even when the balanced truncation method failed.

\section{Accuracy enhancing methods}

A large class of model reduction methods can be interpreted as performing a similarity transformation $Z$ yielding

$$
\left[\begin{array}{c|c}
Z^{-1} A Z & Z^{-1} B \\
\hline C Z & D
\end{array}\right]:=\left[\begin{array}{cc|c}
A_{11} & A_{12} & B_{1} \\
A_{21} & A_{22} & B_{2} \\
\hline C_{1} & C_{2} & D
\end{array}\right],
$$


and then defining the reduced model $G_{r}$ as

$$
\left(A_{r}, B_{r}, C_{r}, D_{r}\right)=\left(A_{11}, B_{1}, C_{1}, D\right) .
$$

When writing

$$
Z:=\left[\begin{array}{cc}
T & U
\end{array}\right], \quad Z^{-1}:=\left[\begin{array}{c}
L \\
V
\end{array}\right],
$$

then $\Pi=T L$ is a projector on $T$ along $L$, and $L T=I_{r}$. Thus the reduced system is given by

$$
\left(A_{r}, B_{r}, C_{r}, D_{r}\right)=(L A T, L B, C T, D) .
$$

The matrices $L$ and $T$ are called truncation matrices.

Partitioned forms as in (1) can also be used to construct a so-called singular perturbation approximation (SPA). The matrices of the reduced model in this case are given by

$$
\begin{aligned}
& A_{r}=A_{11}+A_{12}\left(\gamma I-A_{22}\right)^{-1} A_{21}, \\
& B_{r}=B_{1}+A_{12}\left(\gamma I-A_{22}\right)^{-1} B_{2}, \\
& C_{r}=C_{1}+C_{2}\left(\gamma I-A_{22}\right)^{-1} A_{21}, \\
& D_{r}=D+C_{2}\left(\gamma I-A_{22}\right)^{-1} B_{2} .
\end{aligned}
$$

where $\gamma=0$ for a continuous-time system and $\gamma=1$ for a discrete-time system. Note that SPA formulas preserve the DC-gain of an original stable system.

The emphasis on improving the accuracy of computations has led to so-called algorithms with enhanced accuracy. In many model reduction methods, the matrices $L$ and $T$ are determined from two positive semidefinite matrices $P$ and $Q$, called generically grammians. In general, the grammians satisfy appropriate matrix Lyapunov equations which can be solved directly for the upper triangular Cholesky factors $S$ and $R$ (i.e., $\left.P=S S^{T}, Q=R^{T} R\right)$. The computation of $L$ and $T$ can be done from the singular value decomposition

$$
R S=\left[\begin{array}{ll}
U_{1} & U_{2}
\end{array}\right] \operatorname{diag}\left(\Sigma_{1}, \Sigma_{2}\right)\left[\begin{array}{ll}
V_{1} & V_{2}
\end{array}\right]^{T},
$$

where

$$
\Sigma_{1}=\operatorname{diag}\left(\sigma_{1}, \ldots, \sigma_{r}\right), \quad \Sigma_{2}=\operatorname{diag}\left(\sigma_{r+1}, \ldots, \sigma_{n}\right),
$$

and $\sigma_{1} \geq \ldots \geq \sigma_{r}>\sigma_{r+1} \geq \ldots \geq \sigma_{n} \geq 0$.

The so-called square-root (SR) methods determine $L$ and $T$ as [11]

$$
L=\Sigma_{1}^{-1 / 2} U_{1}^{T} R, \quad T=S V_{1} \Sigma_{1}^{-1 / 2} .
$$

This approach is usually numerically very accurate for well-equilibrated systems. However if the original system is highly unbalanced, potential accuracy losses can be induced in the reduced model if either of the truncation matrices $L$ or $T$ is ill-conditioned (i.e., nearly rank deficient).

To avoid ill-conditioned truncation matrices, a balancing-free (BF) approach has been proposed in
[9] in which $L$ and $T$ are always well-conditioned. A balancing-free square-root (BFSR) algorithm which combines the advantages of the $\mathbf{B F}$ and $\mathbf{S R}$ approaches has been introduced in [13]. $L$ and $T$ are determined as

$$
L=\left(Y^{T} X\right)^{-1} Y^{T}, \quad T=X,
$$

where $X$ and $Y$ are $n \times r$ matrices with orthogonal columns computed from two QR decompositions

$$
S V_{1}=X W, \quad R^{T} U_{1}=Y Z
$$

with $W$ and $Z$ non-singular and upper-triangular. The reduced model obtained in this way is related to that one obtained by the $\mathbf{S R}$ approach by a non-orthogonal state coordinate transformation. Since the accuracy of the BFSR algorithm is usually better than either of $\mathbf{S R}$ or $\mathbf{B F}$ techniques, this approach is the default option in high performance model reduction software [14].

The SPA formulas can be used directly on a balanced minimal order realization of the original system computed with the SR method. A BFSR method to compute SPAs has been proposed in [12]. The essence of this method is to construct truncation matrices $L$ and $T$ such that the system $(L A T, L B, C T, D)$ is a minimal realization of the original system and the product of corresponding grammians has a block-diagonal structure which allows the application of the SPA formulas.

Provided the Cholesky factors $R$ and $S$ are known, the computation of matrices $L$ and $T$ can be done by using exclusively numerically stable algorithms. Even the computation of the singular value decomposition of $R S$ can be done without forming this product. Thus the effectiveness of the $\mathbf{S R}$ or BFSR techniques depends entirely on the accuracy of the computed Cholesky factors of the grammians.

In the unweighted balanced truncation (BT) method proposed originally in [7], $P$ and $Q$ are the standard controllability and observability grammians satisfying a pair of continuous-time (c) or discrete-time (d) Lyapunov equations

$$
\begin{aligned}
& \text { (c) }\left\{\begin{array}{l}
A P+P A^{T}+B B^{T}= \\
A^{T} Q+Q A+C^{T} C=
\end{array}\right. \\
& \text { (d) }\left\{\begin{array}{l}
A P A^{T}+B B^{T}=P \\
A^{T} Q A+C^{T} C=Q
\end{array}\right.
\end{aligned}
$$

These equations can be solved directly for the Cholesky factors of the grammians by using numerically reliable algorithms proposed in [5]. A SR version of the BT method has been proposed in [11] and a BFSR version of this method is described in [13]. A BFSR version of the SPA method is described in [12]. In section 4 we show how to compute the Cholesky factors of frequency-weighted grammians which allow the usage of SR and BFSR techniques for frequency-weighted balancing-related model reduction methods. 


\section{Frequency-weighted balancing related model reduction}

According to the choice of so-called frequency-weighted grammians, two basic approaches are presently used to solve FWMR problems. Each of these approaches can be used in conjunction with either the BT or SPA methods, and the resulting methods can be termed as frequency-weighted balanced truncation (FWBT) and frequency-weighted singular perturbation approximation (FWSPA) methods, respectively. Note that the usage of the SPA method in conjunction with FWMR appears to be new, no mentioning of this method in the literature being known to the authors.

The first FWBT approach has been proposed by Enns in [3]. Assume $G$ and the two weights $W_{o}$ and $W_{i}$ are all stable TFMs, and let $\mathbf{W}_{\mathbf{o}}=\left(A_{o}, B_{o}, C_{o}, D_{o}\right)$ and $\mathbf{W}_{\mathbf{i}}=\left(A_{i}, B_{i}, C_{i}, D_{i}\right)$ be minimal realizations of the weighting matrices. Consider the following realizations of $G W_{i}$ and $W_{o} G$ :

$$
\begin{gathered}
\mathbf{G W}_{\mathbf{i}}=\left[\begin{array}{c|c}
\bar{A}_{i} & \bar{B}_{i} \\
\hline \bar{C}_{i} & \bar{D}_{i}
\end{array}\right]=:\left[\begin{array}{cc|c}
A & B C_{i} & B D_{i} \\
0 & A_{i} & B_{i} \\
\hline C & D C_{i} & D D_{i}
\end{array}\right], \\
\mathbf{W}_{\mathbf{o}} \mathbf{G}=\left[\begin{array}{c|c}
\bar{A}_{o} & \bar{B}_{o} \\
\hline \bar{C}_{o} & \bar{D}_{o}
\end{array}\right]=:\left[\begin{array}{cc|c}
A & 0 & B \\
B_{o} C & A_{o} & B_{o} D \\
\hline D_{o} C & C_{o} & D_{o} D
\end{array}\right]
\end{gathered}
$$

Let

$$
\bar{P}=\left[\begin{array}{ll}
P_{11} & P_{12} \\
P_{12}^{T} & P_{22}
\end{array}\right], \quad \bar{Q}=\left[\begin{array}{ll}
Q_{11} & Q_{12} \\
Q_{12}^{T} & Q_{22}
\end{array}\right],
$$

be the partitioned controllability grammian of $G W_{i}$ and the observability grammian of $W_{o} G$, where $P_{11}$ and $Q_{11}$ are $n \times n$ matrices. The approach proposed by Enns defines

$$
P_{E}=P_{11}, \quad Q_{E}=Q_{11}
$$

as the frequency-weighted controllability and observability grammians, respectively. Although, successfully employed in many application, this method lacks an easily computable a priori error bound and the stability of the reduced model is not guaranteed in the case of two-sided weighting, unless either $W_{o}=I$ or $W_{i}=I$. Moreover, occasionally, quite poor approximations result even for one-sided weighting (see Example 2 in [15]). For supplementary details on this approach see $[1,15]$.

The second approach was proposed in $[6,10]$ and, under certain circumstances, guarantees stability in the case of two-sided weighting. Provided $P_{22}$ and $Q_{22}$ are nonsingular (a condition ensured because the realizations of $W_{o}$ and $W_{i}$ are minimal), the two grammians are chosen as

$$
\begin{aligned}
P_{L} & =P_{11}-P_{12} P_{22}^{-1} P_{12}^{T}, \\
Q_{L} & =Q_{11}-Q_{12} Q_{22}^{-1} Q_{12}^{T}
\end{aligned}
$$

Since $P_{L}$ and $Q_{L}$ satisfy this time Lyapunov equations of the form (3) (but with different $B$ and $C$ matrices), stability is normally automatically guaranteed [8]. As with the Enns' method, no easily computable a priori error bound exists for this approach. The main weakness of this approach is the requirement that no polezero cancellations occur when forming $G W_{i}$ or $W_{o} G$. For example, this restriction prevents the applicability of this method to solve controller reduction problems involving weights such as those introduced in [1].

A modification of the method of Enns which guarantees stability in the case of two-sided weights has been proposed recently by Wang et el. [15] for continuoustime systems. With some preliminary assumptions, this approach allows the computation of an a priori error bound for the weighted approximation error, which in some cases appears to be helpful in guiding the choice of the order of the reduced order model. The two grammians $P_{W}$ and $Q_{W}$ are determined in [15] as the solutions of the appropriate pair of Lyapunov equations

$$
\begin{aligned}
& \text { (c) }\left\{\begin{array}{l}
A P_{W}+P_{W} A^{T}+\widetilde{B} \widetilde{B}^{T}=0 \\
Q_{W} A+A^{T} Q_{W}+\widetilde{C}^{T} \widetilde{C}=0
\end{array}\right. \\
& \text { (d) }\left\{\begin{array}{l}
A P_{W} A^{T}+\widetilde{B} \widetilde{B}^{T}=P_{W} \\
A^{T} Q_{W} A+\widetilde{C}^{T} \widetilde{C}=Q_{W}
\end{array}\right.
\end{aligned}
$$

where $\widetilde{B}$ and $\widetilde{C}$ are fictitious input and output matrices. These matrices are computed from the orthogonal eigendecompositions of the symmetric matrices $X=-A P_{11}-P_{11} A^{T}$ and $Y=-A^{T} Q_{11}-Q_{11} A$ :

$$
X=U \Theta U^{T}, \quad Y=V \Gamma V^{T},
$$

where $\Theta$ and $\Gamma$ are real diagonal matrices. Specifically, according to [15], $\widetilde{B}$ and $\widetilde{C}$ are determined as

$$
\widetilde{B}=U|\Theta|^{\frac{1}{2}}, \quad \widetilde{C}=|\Gamma|^{\frac{1}{2}} V^{T}
$$

where $|\cdot|$ denotes a matrix formed from the absolute values of its elements. Provided $\operatorname{rank}[\widetilde{B} B]=\operatorname{rank} \widetilde{B}$ and $\operatorname{rank}\left[\widetilde{C}^{T} C^{T}\right]=\operatorname{rank} \widetilde{C}^{T}$ it is also proven in $[15]$ that

$$
\left\|W_{o}\left(G-G_{r}\right) W_{i}\right\|_{\infty} \leq k \operatorname{tr} \Sigma_{2}
$$

where $k=2\left\|W_{o} L\right\|_{\infty}\left\|K W_{i}\right\|_{\infty}$ with $L=C \widetilde{C}^{\#}$ and $K=$ $\widetilde{B}^{\#} B\left(X^{\#}\right.$ denotes the pseudo-inverse of $\left.X\right)$. It is easy to see that with this choice of grammians we have $P_{W}-$ $P_{E}=: \Delta P_{W} \geq 0$ and $Q_{W}-Q_{E}=: \Delta Q_{W} \geq 0$. Thus, the system $(A, \widetilde{B}, \widetilde{C})$ is minimal provided the original system is minimal.

It appears that in some cases the sizes of $\Delta P_{W}$ and $\Delta Q_{W}$ can be reduced by another choice of $\widetilde{B}$ and $\widetilde{C}$. Consider $\Theta=\operatorname{diag}\left(\Theta_{1}, \Theta_{2}\right)$ and $\Gamma=\operatorname{diag}\left(\Gamma_{1}, \Gamma_{2}\right)$ in the decompositions of $X$ and $Y$ in (10) partitioned such that $\Theta_{1}>0$ and $\Theta_{2} \leq 0, \Gamma_{1}>0$ and $\Gamma_{2} \leq 0$. Partition 
$U$ and $V$ in accordance with the partitioning of $\Theta$ and $\Gamma$, respectively, and define $\widetilde{B}$ and $\widetilde{C}$ as

$$
\widetilde{B}=U_{1} \Theta_{1}^{\frac{1}{2}}, \quad \widetilde{C}=\Gamma_{1}^{\frac{1}{2}} V_{1}^{T} .
$$

With this choice, provided similar rank conditions are fulfilled, an error bound as in (11) holds with a smaller $k$ ( $L$ and $K$ have smaller norms) and a possibly smaller norm $\Sigma_{2}$.

It is possible to construct frequency-weighted grammians which, in a way, combines the approaches of $[6,10]$ with Enns' method [3]. In a combination method we can use as frequency-weighted grammians

$$
\begin{aligned}
P_{E L} & =P_{11}-\alpha_{c}^{2} P_{12} P_{22}^{-1} P_{12}^{T}, \\
Q_{E L} & =Q_{11}-\alpha_{o}^{2} Q_{12} Q_{22}^{-1} Q_{12}^{T}
\end{aligned}
$$

where for $\alpha_{c}=\alpha_{o}=0$ we have the choice for Enns' method, while for $\alpha_{c}=\alpha_{o}=1$ we have the choice for the method of [6] with stability guarantee. Because stability is guaranteed for $\alpha_{c}=\alpha_{o}=1$, it is to be expected this to be also true for nearby subunitary values of $\alpha_{c}$ and $\alpha_{o}$ (on the basis of a continuous variation of spectrum with $\alpha_{c}$ and $\alpha_{o}$ ). Thus, stability will be guaranteed in a whole neighborhood of $\alpha_{c}=\alpha_{o}=1$ regardless of whether pole-zero cancellations occur. This feature can be seen as a simultaneous enhancement of each of the methods in $[3,6]$.

Another way to ensure stability of reduced models in the case of two-sided weights for all subunitary values of parameters $\alpha_{c}$ and $\alpha_{o}$, is to use a modified combination method, where we apply the modified method of Enns to the grammians used in the combination method. This can be done by defining new fictitious input and output matrices $\widehat{B}$ and $\widehat{C}$, respectively, such that the frequency-weighted grammians $P_{V}$ and $Q_{V}$ satisfy

$$
\begin{aligned}
& \text { (c) }\left\{\begin{array}{l}
A P_{V}+P_{V} A^{T}+\widetilde{B} \widetilde{B}^{T}=0 \\
Q_{V} A+A^{T} Q_{V}+\widetilde{C}^{T} \widetilde{C}=0
\end{array}\right. \\
& (d)\left\{\begin{array}{l}
A P_{V} A^{T}+\widetilde{B} \widetilde{B}^{T}=P_{V} \\
A^{T} Q_{V} A+\widetilde{C}^{T} \widetilde{C}=Q_{V}
\end{array}\right.
\end{aligned}
$$

$\widetilde{B}$ and $\widetilde{C}$ are defined as in (12) using the decompositions (10) with

$$
\begin{aligned}
& \text { (c) }\left\{\begin{array}{l}
X=-A P_{E L}-P_{E L} A^{T} \\
Y=-A^{T} Q_{E L}-Q_{E L} A
\end{array}\right. \\
& \text { (d) }\left\{\begin{array}{l}
X=-A P_{E L} A^{T}+P_{E L} \\
Y=-A^{T} Q_{E L} A+Q_{E L}
\end{array}\right.
\end{aligned}
$$

Note that any combination of grammians $\left(P_{E L}, Q_{V}\right)$, $\left(P_{V}, Q_{E L}\right)$, or $\left(P_{V}, Q_{V}\right)$ guarantees the stability of approximations for two-sided weighting.

\section{Square-root techniques}

In this section we show how square-root formulas can be employed to compute the grammian matrices for the specific choices described in the previous section. Assume $\bar{S}$ and $\bar{R}$ are the Cholesky factors of $\bar{P}$ and $\bar{Q}$ in (6), respectively, satisfying $\bar{P}=\bar{S} \bar{S}^{T}$ and $\bar{Q}=\bar{R}^{T} \bar{R}$. These factors can be computed using the method of Hammarling [5] to solve directly for the Cholesky factors the appropriate continuous-time (c) or discretetime (d) Lyapunov equations

$$
\begin{aligned}
& \text { (c) }\left\{\begin{array}{l}
\bar{A}_{i} \bar{P}+\bar{P} \bar{A}_{i}^{T}+\bar{B}_{i} \bar{B}_{i}^{T}=0 \\
\bar{A}_{o}^{T} \bar{Q}+\bar{Q} \bar{A}_{o}+\bar{C}_{o}^{T} \bar{C}_{o}=0
\end{array}\right. \\
& \text { (d) }\left\{\begin{array}{l}
\bar{A}_{i} \bar{P} \bar{A}_{i}^{T}+\bar{B}_{i} \bar{B}_{i}^{T}=\bar{P} \\
\bar{A}_{o}^{T} \bar{Q} \bar{A}_{o}+\bar{C}_{o}^{T} \bar{C}_{o}=\bar{Q}
\end{array}\right.
\end{aligned}
$$

The solution of these Lyapunov equations involves the reduction of each of matrices $\bar{A}_{i}$ and $\bar{A}_{o}$ to a real Schur form (RSF). For efficiency reasons the reduction of $A$, $A_{i}$ and $A_{o}$ to RSF is preferable to be done independently and only once. Therefore, for computational convenience we prefer to work with an alternative (permuted) realization of $W_{o} G$

$$
\mathbf{W}_{\mathbf{o}} \mathbf{G}=\left[\begin{array}{c|c}
\bar{A}_{o} & \bar{B}_{o} \\
\hline \bar{C}_{o} & \bar{D}_{o}
\end{array}\right]=:\left[\begin{array}{cc|c}
A_{o} & B_{o} C & B_{o} D \\
0 & A & B \\
\hline C_{o} & D_{o} C & D_{o} D
\end{array}\right]
$$

With the realization (4) of $G W_{i}$ and with the realization (14) of $W_{o} G, \bar{A}_{i}$ and $\bar{A}_{o}$ are in RSF if $A, A_{i}$ and $A_{o}$ are in RSF. Note that the observability gramian $\bar{Q}$ corresponding to realization (14) has the permuted block structure

$$
\bar{Q}=\left[\begin{array}{ll}
Q_{22} & Q_{12}^{T} \\
Q_{12} & Q_{11}
\end{array}\right]
$$

(compare this with $\bar{Q}$ defined in (6)).

If we partition $\bar{S}$ and $\bar{R}$ as

$$
\bar{S}=\left[\begin{array}{cc}
S_{11} & S_{12} \\
0 & S_{22}
\end{array}\right], \quad \bar{R}=\left[\begin{array}{cc}
R_{11} & R_{12} \\
0 & R_{22}
\end{array}\right]
$$

we have immediately that the Cholesky factors of $P_{E}=S_{E} S_{E}^{T}$ and $Q_{E}=R_{E}^{T} R_{E}$ corresponding to Enns' method are given by

$S_{E} S_{E}^{T}=S_{11} S_{11}^{T}+S_{12} S_{12}^{T}, \quad R_{E}^{T} R_{E}=R_{22}^{T} R_{22}+R_{12}^{T} R_{12}$.

Thus, to compute $S_{E}$ the RQ-factorization of the matrix $\left[\begin{array}{ll}S_{11} & S_{12}\end{array}\right]$ must be performed, while for computing $R_{E}$ the QR-factorization of $\left[\begin{array}{l}R_{22} \\ R_{12}\end{array}\right]$ must be performed. Both these factorizations can be computed using well established factorization updating techniques [4] which fully exploit the upper triangular shapes of $S_{11}$ and $R_{22}$. 
For the Cholesky factors of $P_{L}=S_{L} S_{L}^{T}$ and $Q_{L}=$ $R_{L}^{T} R_{L}$ corresponding to the method of [6] we simply have

$$
S_{L}=S_{11}, \quad R_{L}=R_{22} .
$$

Thus, by working directly with the Cholesky factors we completely avoid the matrix inversions necessary when forming $P_{L}$ and $Q_{L}$ directly from (8). Therefore, no difficulties are expected to be encountered when $P_{22}=$ $S_{22} S_{22}^{T}$ or $Q_{22}=R_{11}^{T} R_{11}$ are ill-conditioned or even exactly singular (i.e., the state space representations of $W_{i}$ and/or $W_{o}$ are not minimal).

For the combined problem, the Cholesky factors of $P_{E L}=S_{E L} S_{E L}^{T}$ and $Q_{E L}=R_{E L}^{T} R_{E L}$ are given by

$$
\begin{aligned}
& S_{E L} S_{E L}^{T}=S_{11} S_{11}^{T}+\left(1-\alpha_{c}^{2}\right) S_{12} S_{12}^{T} \\
& R_{E L}^{T} S_{E L}=R_{22}^{T} R_{22}+\left(1-\alpha_{o}^{2}\right) R_{12}^{T} R_{12}
\end{aligned}
$$

and can be computed from the RQ-decomposition of $\left[\begin{array}{ll}S_{11} & \sqrt{1-\alpha_{c}^{2}} S_{12}\end{array}\right]$ and QR-decomposition of $\left[\frac{R_{22}}{\sqrt{1-\alpha_{o}^{2}} R_{12}}\right]$.

For the modified method, the Cholesky factors of $P_{V}=$ $S_{V} S_{V}^{T}$ and $Q_{V}=R_{V}^{T} R_{V}$ result by solving (13) directly for these factors using the algorithm of Hammarling [5].

Having the Cholesky-factors for the frequency-weighted grammians, we can compute in a numerical reliable way the truncation matrices $L$ and $T$ using either the SR or BFSR technique. The new square-root formulations of both the FWBT and FWSPA methods are entirely satisfactory from a numerical point of view and can serve as a basis for robust software implementations of the frequency-weighted balancing related approach.

\section{Numerical examples}

Example 1. This example, taken from [10], shows that the FWBT method of Enns may yield unstable reduced order models. The original continuous-time system has the TFM

$$
G(s)=\frac{8 s^{2}+6 s+2}{s^{3}+4 s^{2}+5 s+2}
$$

and the input and output weights are

$$
W_{i}=\frac{1}{s+3}, \quad W_{o}=\frac{1}{s+4} .
$$

The frequency weighted Hankel singular values for the Enns' method are $\{0.0513,0.0417,0.0057\}$. The first order approximation computed with the Enns' method gives an unstable model [10]. However, Enns' method used in conjunction with the SPA method gives

$$
G_{r}=\frac{2.398 s+1.739}{s+1.739}
$$

with the error norm is $\left\|W_{o}\left(G-G_{r}\right) W_{i}\right\|_{\infty}=0.0855$. Stability has been also achieved by using the combination method for all values of $\alpha_{c}$ and $\alpha_{o}$ satisfying $0.7 \leq \alpha_{c} \leq 1$ and $0.7 \leq \alpha_{o} \leq 1$.

Since the weights vanish at $s=\infty$, forcing $G(s)$ to be exactly matched by its approximation at $s=\infty$ is in a sense wasteful. Indeed, the weights assume their greatest magnitude at $s=0$, and SPA forces an exact match at $s=0$, and presumably a good match near this point. It is hardly surprising for this problem that a better result is achieved with SPA.

Example 2. This is a discrete-time example from [10]

$$
G(z)=\frac{z^{3}}{z^{4}+1.1 z^{3}-0.01 z^{2}-0.275 z-0.06}
$$

with the weights

$$
W_{i}(z)=W_{o}(z)=\frac{z+0.9}{z+0.1}
$$

The frequency weighted Hankel singular values for Enns' method are $\{1.1439,0.3106,0.2391,0.0032\}$. The first order approximation computed with the discrete-time version of the FWBT method of Enns gives an unstable reduced model [10]. Once again the FWSPA method gives a stable reduced model

$$
G_{r}=\frac{-0.00188 z+1.073}{z+0.8796}
$$

fulfilling a very good approximation error bound $\left\|W_{o}\left(G-G_{r}\right) W_{i}\right\|_{\infty}=0.4812$.

Example 3. This is the fourth order model used in [10] with the frequency weights

$$
\mathbf{W}_{\mathbf{i}}=\mathbf{W}_{\mathbf{o}}=\left(-4.5 I_{2}, 3 I_{2}, 1.5 I_{2}, I_{2}\right) .
$$

We performed reductions to orders $r$ of $G_{r} 3,2$ and 1 using the combination method taking as values of $\alpha=\alpha_{c}=\alpha_{o} 0,0.5$ and 1 . Recall that the value $\alpha=0$ corresponds to Enns' method while $\alpha=1$ corresponds to the method of [6]. In each case we computed the weighted approximation error $\left\|W_{o}\left(G-G_{r}\right) W_{i}\right\|_{\infty}$ for both the FWBT and FWSPA methods. The results are given in Table 1, where for comparison purposes, we have added in the last column the results reported for the method proposed in [15].

\begin{tabular}{|c||c|c|c||c|c|c||c|}
\hline & \multicolumn{3}{c||}{ FWBT } & \multicolumn{3}{c||}{ FWSPA } & {$[15]$} \\
\hline$r$ & $\alpha=0$ & $\alpha=0.5$ & $\alpha=1$ & $\alpha=0$ & $\alpha=0.5$ & $\alpha=1$ & \\
\hline 1 & 2.112 & 2.116 & 2.566 & $\mathbf{1 . 4 0 5}$ & 1.495 & 2.035 & 2.121 \\
2 & 0.265 & 0.261 & 0.560 & $\mathbf{0 . 2 5 0}$ & 0.256 & 0.687 & 0.272 \\
3 & 0.112 & 0.110 & 0.164 & $\mathbf{0 . 0 6 5}$ & 0.069 & 0.121 & 0.115 \\
\hline
\end{tabular}

Table 1: Weighted errors for the FWBT, FWSPA and method of Wang et al. [15] 


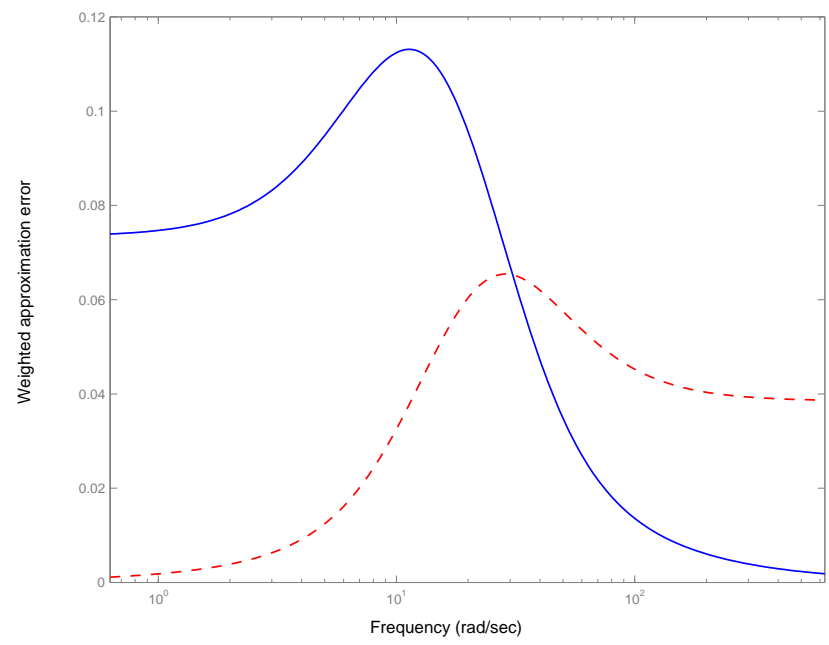

Figure 1: Weighted approximation error $\left\|W_{o}(j \omega)\left(G(j \omega)-G_{r}(j \omega)\right) W_{i}(j \omega)\right\|_{\infty}: \quad$ FWBT (solid), FWSPA (dashed).

The modified combination method, with $\alpha=0$ and the pair $\left(P_{V}, Q_{V}\right)$ computed as in (13), leads for both FWBT and FWSPA to almost the same errors as those in Table 1. In all cases the errors are smaller than those for the method of [15].

The FWSPA method for $\alpha=0$ (see values in bold face) behaves uniformly better on this example than all other approaches. We postulate that the good behaviour is probably due to the fact that, by forcing a zero error in the steady-state gain, lower error results also in the low frequency domain which partly overlaps with the frequency region where the weights are most active. This can be also seen looking at Figure 1, where the weighted error norms for the FWBT and FWSPA methods are shown for the 3-rd order approximations.

\section{Conclusions}

We have proposed several new choices for frequencyweighted controllability and observability grammians which enlarge substantially the applicability of frequency-weighted balancing related model reduction methods. To guarantee the stability of the reduced order model in the case of two-sided weighting, modified approaches for Enns' method and a new combination method have been proposed. Although the use of the SPA method in conjunction with frequency-weighted balancing is apparently not even mentioned in the literature, surprisingly this approach provides, almost always, better approximations than the BT method, both in terms of preserving stability as well as leading to lower approximation errors. For the newly developed methods, robust numerical software is available in the FORTRAN 77 library SLICOT [2], together with user friendly MATLAB interfaces.

\section{References}

[1] B. D. O. Anderson and Y. Liu. Controller reduction: concepts and approaches. IEEE Trans. Autom. Control, 34:802-812, 1989.

[2] P. Benner, V. Mehrmann, V. Sima, S. Van Huffel, and A. Varga. SLICOT - a subroutine library in systems and control theory. In B. N. Datta, editor, $A p$ plied and Computational Control, Signals and Circuits, vol. 1, pp. 499-539. Birkhäuser, 1999.

[3] D. Enns. Model Reduction for Control Systems Design. PhD thesis, Dept. Aeronaut. Astronaut., Stanford Univ., Stanford, CA, 1984.

[4] P. E. Gill, G. H. Golub, W. Murray, and M. A. Saunders. Methods for modifying matrix factorizations. Math. Comput., 28:505-535, 1974.

[5] S. J. Hammarling. Numerical solution of the stable, non-negative definite Lyapunov equation. IMA J. Numer. Anal., 2:303-323, 1982.

[6] C.-A. Lin and T.-Y. Chiu. Model reduction via frequency weighted balanced realization. CONTROL Theory and Advanced Technology, 8:341-351, 1992.

[7] B. C. Moore. Principal component analysis in linear system: controllability, observability and model reduction. IEEE Trans. Autom. Control, 26:17-32, 1981.

[8] L. Pernebo and L. M. Silverman. Model reduction via balanced state space representations. IEEE Trans. Autom. Control, 27:382-387, 1982.

[9] M. G. Safonov and R. Y. Chiang. A Schur method for balanced-truncation model reduction. IEEE Trans. Autom. Control, 34:729-733, 1989.

[10] V. Sreeram, B.D.O. Anderson, and A.G. Madievski. New results on frequency weighted balanced reduction technique. In Proc. 1995 ACC, Seattle, WA, pp. 4004-4009, 1995.

[11] M. S. Tombs and I. Postlethwaite. Truncated balanced realization of a stable non-minimal state-space system. Int. J. Control, 46:1319-1330, 1987.

[12] A. Varga. Balancing-free square-root algorithm for computing singular perturbation approximations. In Proc. of 30th IEEE CDC, Brighton, UK, pp. 10621065, 1991.

[13] A. Varga. Efficient minimal realization procedure based on balancing. In A. El Moudni, P. Borne, and S. G. Tzafestas, editors, Prepr. of IMACS Symp. on Modelling and Control of Technological Systems, vol. 2, pp. 42-47, 1991.

[14] A. Varga. Model reduction software in the SLICOT library. In B. N. Datta, editor, Applied and Computational Control, Signals and Circuits, vol. 2, Birkhäuser, 2001 (to appear).

[15] G. Wang, V. Sreeram, and W. Q. Liu. A new frequency-weighted balanced truncation method and error bound. IEEE Trans. Autom. Control, 44:17341737, 1999. 